MODELING, IDENTIFICATION AND CONTROL, 1993, VOL. 14, NO. 2, 73-84

doi:10.4173/mic.1993.22

\title{
Catalytic cracking models developed for predictive control purposes
}

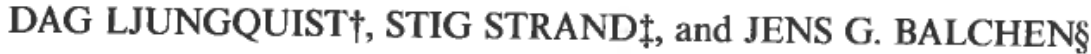
Keywords: State-space methods, catalytic cracking, predictive control, modeling,
model validation,

\begin{abstract}
The paper deals with state-space modeling issues in the context of model-predictive control, with application to catalytic cracking. Emphasis is placed on model establishment, verification and online adjustment. Both the Fluid Catalytic Cracking (FCC) and the Residual Catalytic Cracking (RCC) units are discussed. Catalytic cracking units involve complex interactive processes which are difficult to operate and control in an economically optimal way. The strong nonlinearities of the FCC process mean that the control calculation should be based on a nonlinear model with the relevant constraints included. However, the model can be simple compared to the complexity of the catalytic cracking plant. Model validity is ensured by a robust online model adjustment strategy. Model-predictive control schemes based on linear convolution models have been successfully applied to the supervisory dynamic control of catalytic cracking units, and the control can be further improved by the SSPC scheme.
\end{abstract}

\section{Introduction}

It has long been recognized that model-predictive control (MPC) strategies meet most of the supervisory controller requirements of the process industries. In the presence of quality constraints on manipulated independent process variables, MPC is in fact the only generally successful concept.

Model-based and optimal control have a long history, but were infrequently used in the process industries until Cutler and Ramaker (1979) presented their Dynamic Matrix Control (DMC), while Richalet et al. (1978) came up with a very similar version of model-predictive control. One major later contribution was QDMC (Garcia and Morshedi, 1986), which treats constraints in a direct and natural manner.

A lot of successful implementations based on these and other MPC algorithms using linear input-output dynamic models with constraints have been reported. Successful in the sense that they perform much better than the replaced controllers because they provide the more economical process operation at, or close to, the intersection of constraints.

However, the linear experimental models generally have poor long-range prediction capabilities when the process operating conditions are drastically changed, so that nonlinear process dynamics is pronounced. Such severe changes are the natural results of large process disturbances and set-point changes, to which the MPC is forced to respond.

Received 10 June 1992

$\dagger$ Hydro Aluminium a.s, Årdal Verk N-5870 Øvre Årdal, Norway.

† SINTEF Automatic Control N-7034 Trondheim, Norway.

§Division of Engineering Cybernetics, The Norwegian Institute of Technology N-7034 Trondheim, Norway.

Reprinted from Proceedings of DYCORD + 92, College Park, Maryland, April 1992, with permission from Pergamon Press. 
As will be argued in this paper, an MPC based on a physically established statespace model generally has the desired prediction capability. This has been recognized by several authors (e.g. Jang et al., 1987; Joseph et al., 1988; Patwardhan et al., 1988; Sistu and Bequette, 1990; Schmid and Biegler, 1990). Recently the State-Space Predictive Control (SSPC) scheme has been developed based on these model considerations (Balchen et al., 1988a, 1988b, 1991; Ljungquist, 1990; Strand, 1991).

The modeling aspects of predictive control are highlighted in the following sections. The presentation starts with a summary of the features of the state-space model which make it ideally suited for predictive control purposes. Model verification and adjustment strategies are also addressed. Then catalytic cracking models developed for predictive control purposes will be discussed. Both the Fluid Catalytic Cracker (FCC) and the more complex Residual Catalytic Cracker (RCC) are described.

\section{State-space modeling}

Reliable process models form the basis for the development of predictive control schemes because model-predictive control relies heavily on the predictive capabilities of the model. Ljungquist (1990) pinpoints that the nonlinear state-space model possesses a number of advantages which are important to the flexibility of the resulting predictive controllers:

- The model building can be based on 'first principles' which means that a priori information is included in the model in a natural way.

- The predictive capabilities are good when the model structure is correct.

- The state variables generally represent physical quantities.

- Nonlinearities are easily expressed.

- Constraints on any process variable can be included in the model.

- The model is resetable because the states and the model structure contain all the process history.

The system identification procedure which generally must be executed to establish a model with satisfactory online predictive capabilities are summarized in Fig. 1. Clarification of the model application area is a critical part of the conceptual modeling. It is well-known from the control theory that a correct model structure around the bandwidth frequency is very important to the performance of all model-based controllers, while the steady-state predictive capabilities usually are less important. This holds true for the SSPC as well. It should be noted however, that an SSPC strategy based on an economic performance index can only reach the optimal operation point when the steady-state prediction of the model is correct. Integral action towards the optimal steady state can only be achieved by means of a carefully implemented estimation scheme. In an SSPC strategy based on a set-point criterion integral action towards the set points can be included in the control calculations.

A correct model structure is essential to obtain reliable predictions. The model complexity and the mathematical formulation of the model are central factors. As listed above the state-space model is a very convenient formulation. Physical insight can then be used to locate and eliminate weak interactions and very slow or very fast dynamics. Usually some phenomena of the process are poorly known and extensive offline experiments, including offline parameter estimation, must be performed to arrive at a satisfactory model. All dynamic variables which are important to the performance index and constraints should be included in the model as state variables. 


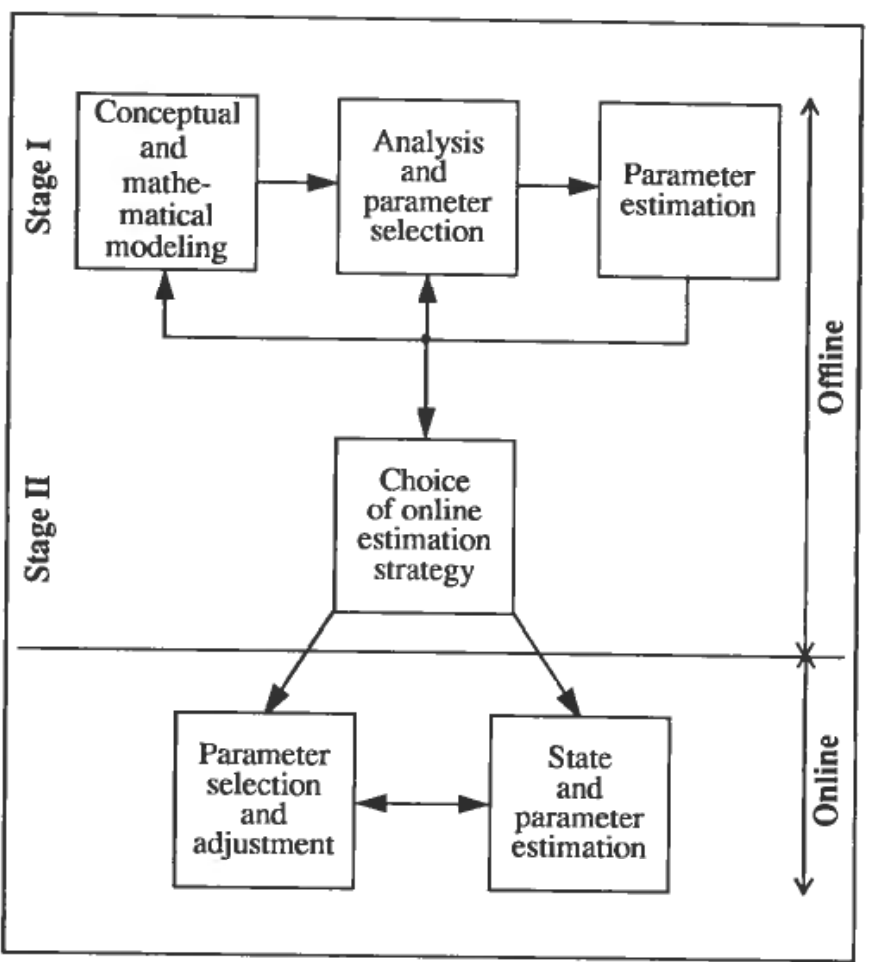

Figure 1. A typical system identification procedure.

It is easy to tune an over-parametrized model to give satisfactory short-range predictions, but the long-range predictive capabilities will usually be very poor. This fact is closely related to identifiability. It can be shown (Ljungquist, 1990) that the state covariance matrix (or equivalently the measurement covariance in other model formulations) is proportional to the covariance of the parameter vector:

$$
X \sim \frac{\mathrm{d} x}{\mathrm{~d} \theta} \Theta \frac{\mathrm{d} x^{\mathrm{T}}}{\mathrm{d} \theta}
$$

where

$X$ is the state covariance matrix

$x$ is the state variable vector

$\Theta$ is the parameter covariance matrix

$\theta$ is the parameter vector

Assume that a robust estimation algorithm is applied to estimate a set of poorly identifiable parameters. Although the parameter values do not diverge, the covariance matrix elements will be very large. Despite these large values an acceptable $a$ posteriori state covariance matrix can be obtained if a state update matrix is included in the estimator. Consequently, acceptable short-range predictions can be achieved even though the parameter estimates are highly biased. However, this does not hold for longrange predictions in general. In fact it is more important to ensure that the identifiability of the parameter set is acceptable than to ensure that all the timevarying parameters are included in the estimated parameter set.

The insight gained from the modeling provides valuable experience which can be used when the online model adjustment strategy is to be derived. Different adjustment 
strategies to be used in SSPC schemes are discussed by Ljungquist (1990). The state variables of the model have to be updated online in order to keep the prediction errors at acceptable levels. Some of the model parameters can be adjusted according to a data base. The model sensitivity to parameter uncertainty is different for the various parameters. If this sensitivity is high compared to the parameter variations and the parameters are not available from a data base, an online parameter estimation scheme must be applied. Moreover, parameters which vary frequently should also be estimated online. As indicated above, the parameter identifiability restricts the number of parameters or parameter combinations which can be estimated simultaneously. This number depends on the estimation scheme and the a priori information on parameter variation. Since the parameter identifiablity depends heavily on the process excitations, it may be necessary to implement a parameter selector online to ensure parameter identifiability.

\section{Fluid catalytic cracking}

The Fluid Catalytic Cracker (FCC) produces light hydrocarbons, mainly gasoline, from a heavy feedstock, and it is perhaps the most important process unit in the oil refinery and yields the largest benefit from advanced control. The process is highly nonlinear with very strong cross couplings which means that a constant ordinary control algorithm does not perform satisfactorily. Moreover, $\mathrm{FCC}$ units are required to run under different operating modes due to changes in the refinery feedstocks, changing economics, market demand for products and mechanical constraints. There is significant economic incentive to operate close to constraints, but this cannot easily be achieved by conventional control schemes due to the complexity of the FCC process. Since dynamic models which seem to reproduce the dominating characteristics are reported, the FCC unit is an excellent candidate for realizing substantial benefits with the SSPC.

\subsection{Process characteristics}

A modern FCC process consists mainly of three parts, a riser, a separator and a regenerator as illustrated in Fig. 2. The preheated heavy feed oil is mixed with hot catalyst from the regenerator and reacts endothermically in the riser to form lighter hydrocarbons as well as coke. The lighter hydrocarbons, mainly gasoline, are separated from the catalyst in the separator and sent to the fractionator, while the coke is deposited on the catalyst which is returned to the regenerator. In order to maintain an acceptable catalyst activity the coke is burned off in the regenerator. The air needed for this combustion is supplied at the bottom of the regenerator in such a way that the catalyst is kept fluidized in the rising air. This ensures good air and catalyst mixing in the so called dense bed of the regenerator. Irreversible deactivation and loss of catalyst in the flue gas mean that makeup catalyst and catalyst withdrawal are needed during continuous operation of the FCC unit.

\subsection{FCC modeling}

A review of the dynamic FCC models presented in the open literature can be found in Ljungquist (1990). He concludes that the model proposed by Lee and Groves (1985) is adequate for predictive control purposes because the main two objectives of a simple FCC model are a reasonable description of the regenerator dynamics and a reasonable prediction of the gasoline yield. Lee and Groves' model is quasi-dynamic because the riser equations are steady-state ordinary differential equations (ODEs) which are 


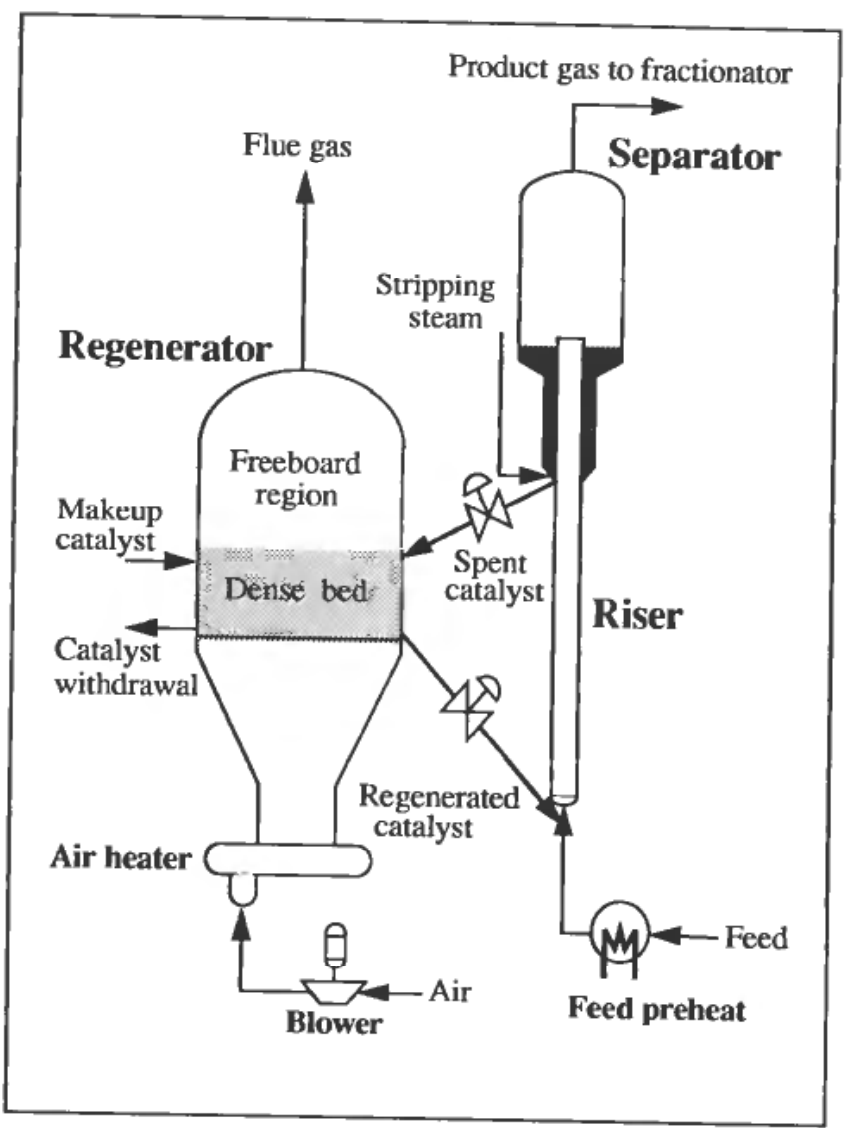

Figure 2. Schematic diagram of a cracking plant.

solved every 0.5 minute, while the regenerator is modelled using dynamic energy and mass balances. The resulting model has three state variables (regenerator dense bed temperature and oxygen fraction, and coke on regenerated catalyst) and the largest time constant is approximately ten thousand times the smallest time constant. Numerical simulation of the model is consequently very time consuming. The analysis performed by Ljungquist (1990) resulted in modifications of the model proposed by Lee and Groves which reduce the simulation requirements drastically as summarized below.

The static riser ODEs can be replaced by a simple algebraic bootstrap model with negligible error impact on the predicted riser yields. This simplifies model analysis. The simulation costs are also reduced, although removal of the smallest time constant is much more important to this improvement; analysis shows that the smallest time constant is closely related to the oxygen balance in the regenerator dense bed:

$$
W_{a} \frac{d O_{d}}{d t}=R_{a}\left(O_{i n}-O_{d}\right)-\frac{n+2+(n+4) \sigma}{4 M_{c}(1+\sigma)} k O_{d} C_{r c} W
$$

where

$W_{a}$ air holdup in regenerator dense bed

$O_{d}$ oxygen mole fraction in regenerator dense bed

$R_{a}$ molar flow rate of air to regenerator 
$O_{i n}$ oxygen mole fraction in air

$n$ hydrogen content in coke

$M_{c}$ molecular weight of coke

$\sigma \quad \mathrm{CO}_{2} / \mathrm{CO}$ ratio in regenerator dense bed

$k$ reaction rate of coke combustion

$C_{r c}$ weight fraction of coke on regenerated catalyst

$W$ catalyst holdup in regenerator dense bed

Removal of this time constant by replacing (1) by the corresponding steady-state solution

$$
O_{d}=\frac{R_{a} O_{i n}}{R_{a}+\frac{n+2+(n+4) \sigma}{4 M_{c}(1+\sigma)} k C_{r c} W}
$$

has negligible effect on the model responses around the bandwidth frequency. As a result the simulation costs are reduced almost by a factor of 100 . Alternatively, if the implemented model has incorrect order around the bandwidth frequency compared to the FCC plant under consideration, $W_{a}$ can be increased to an artificial value and thereby both reducing the stiffness and increasing the model order around the bandwidth frequency. This will be an alternative to the development of a more complex regenerator model.

In the modified model proposed by Ljungquist (1990) simple afterburning and catalyst consumption models are included. The reason is that these quantities are essential to the formulation of the performance index on which the control calculation is based. In summary, it is believed that the proposed model contains the dominating dynamic characteristics. However, it has to be extended to include all the variables which are necessary to take all the constraints on a real FCC unit into account and to formulate a performance index which takes full advantage of the economic incentives of the SSPC flexibility.

Defining the constraints and the performance index on which the computations are based is an important part of the modeling procedure. In summary there are two reasons why the nonlinear state-space model is the only way to represent the FCC sufficiently well for predictive control purposes:

- The nonlinearity of the dynamics are predominant;

- There are internal variables which cannot be measured online and which are constrained and/or important to the performance index. These variables have to be included in the model.

The high nonlinearity means that a nonlinear model must be used and that it is difficult to establish a nonlinear black-box model with reliable long-range prediction capabilities. To illustrate this, the regenerator temperature response to a change in the catalyst circulation rate exhibits inverse dynamics only at some operational points, and the response to a change in the coke formation rate is highly dependent both of the sign of the change and the operational point.

As an example of an alternative modeling approach, neural networks are claimed to be able to model any nonlinear function (Hornik et al., 1989; Poggio and Girosi, 1990). It remains to be proven however, whether such a description can be used to successfully predict the dynamic behaviour of a highly nonlinear process by including past input and output variables in the input layer of the network. Clearly, the number of nodes will have to be very high and it will be an extremely difficult task to train the network to give 
satisfactory predictions under various conditions. This, and the fact that internal process variables cannot be represented and updated online by a neural network representation, mean that such a model cannot be used for predictive control purposes for the FCC.

\subsection{Online model adjustment strategies}

Model analysis and simulation studies have been carried through to examine the ability of different estimation schemes to detect changes in an FCC process and adjust the model accordingly. Recursive methods such as the extended Kalman filter and recursive formulations of a prediction error method based on a maximum likelihood criterion have successfully been implemented. In addition, simulation studies of state and parameter estimation based on more robust but computationally more expensive algorithms, denoted moving-horizon batch methods, have been performed. Although the simulation experiments are performed under idealistic conditions compared to practical FCC process/model matching, it can be concluded that there are estimation schemes which can adjust the model in a satisfactory way provided that the model structure represents the real physical phenomena in a reasonable manner. Moreover, the required computing capacity is available today at an acceptable cost.

Since the FCC process is very complex and data bases are available for conventional FCC operation, it is believed that state updating combined with a data base parameter adjustment strategy can provide sufficiently satisfactory predictive capabilities for a model to be used in the SSPC. On the other hand, the disturbance frequency is so high that an on line parameter estimation scheme will be advantageous. If inclusion of a parameter estimation scheme is considered, the number of parameters should be kept to a minimum to guarantee identifiability of the estimated parameters. This means that the estimated parameters must be selected with care. A movinghorizon batch method should be used if it turns out that more than four parameters have to be estimated online. Otherwise a recursive estimation algorithm can be used.

\subsection{FCC control}

A short review of the FCC control strategies reported up to 1980 can be found in Balchen and Mummé (1988). Those academic exercises concentrated on the complex dynamics due to strong cross-couplings between the endothermic riser and the exothermic regenerator sections and nonlinearities in the reaction rates. The applied FCC models stressed these effects, as is also true for the model discussed here. The feed temperature and the temperature of the air to the regenerator are set to constant values while the mass flow rate of spent catalyst is used to control the mass holdup in the stripper section. This leaves two variables free for manipulation, the flow rate of regenerated catalyst and the flow rate of air to the regenerator. The controlled properties coincide with the three measurements: riser outlet temperature, regenerator temperature and the oxygen concentration of the dense bed.

The performance of the SSPC scheme is compared to conventional single-loop PID-controllers with different pairing in Balchen et al. (1992), with respect to reduction of the dynamic interactions at an unconstrained operating point. The preference to SSPC is quite clear.

The supervisory control of a real FCC is much more complex than can be demonstrated by the present model. This is clearly shown by Caldwell and Dearwater 
(1991), who report successful MPC implementation on six FCC units. They divide the constraints in three groups:

- Maximum gas make constraints;

- maximum wet gas compressor loading

- maximum gas plant loading

- maximum fractionator pressure

- Maximum throughput constraints;

- main fractionator flooding

- maximum reactor or regenerator pressures due to hydraulic pressure buildup

- Maximum coke burning constraints;

- maximum regenerator temperature limits

- minimum regenerator flue gas $\% \mathrm{O}_{2}$ in complete combustion mode or maximum regenerator flue gas $\% \mathrm{CO}$ in partial combustion mode

- maximum regenerator air rate

They also list the manipulated variables:

- air flow rate to the regenerator

- riser outlet temperature

- fresh feed rate

- recycle feed rates

- feed temperatures

The riser outlet temperature (set point) is defined as a manipulated variable for the supervisory control because there is a lower level control loop from the riser outlet temperature measurement to the regenerated catalyst valve.

It can be added that the maximum regenerator air rate will depend on the regenerator pressure due to pressure-dependent compressor capacities.

Clearly, the model discussed here does not include all the effects needed for a valuable performance comparison of different MCP strategies. The paper of McFarlane et al. (1990) presents an FCC model which can serve as such a test-bench, by including a rigorous main fractionator model with recycles to the FCC feed.

The potential benefits from using nonlinear models are indicated through simulations by Schmid and Biegler (1990) and Huang et al. (1989), and the constraints are so predominant that state-space models should be used to fully utilize the potentials of a predictive controller. Nevertheless, the reported MPC implementations are based on input-output linear model dynamics (Prett and Gillette, 1979; Caldwell and Dearwater, 1991).

\section{Residual catalytic cracking}

UOP's Residual Catalytic Cracking (RCC) unit makes it possible to improve refinery for direct conversion of residual feedstocks into transportation fuels and other lighter products. RCC unit are more complex than the FCC, but despite the complexity, RCC units seem to be easier to control than the FCC process. However, RCC dynamics alters drastically as the operational point changes. As a consequence, SSPC will be advantageous because the RCC unit can then be operated at points where conventional control schemes fail. This section is based on papers by Zandona et al. (1982) and Hemler et al. (1984), and the United States Patent no. 4,849,091 (1989). A model based on these references and the paper by Lee and Groves (1985) is presented by Ljungquist (1990). Some of the numerical values are motivated by the paper by Huang et al. (1989). 


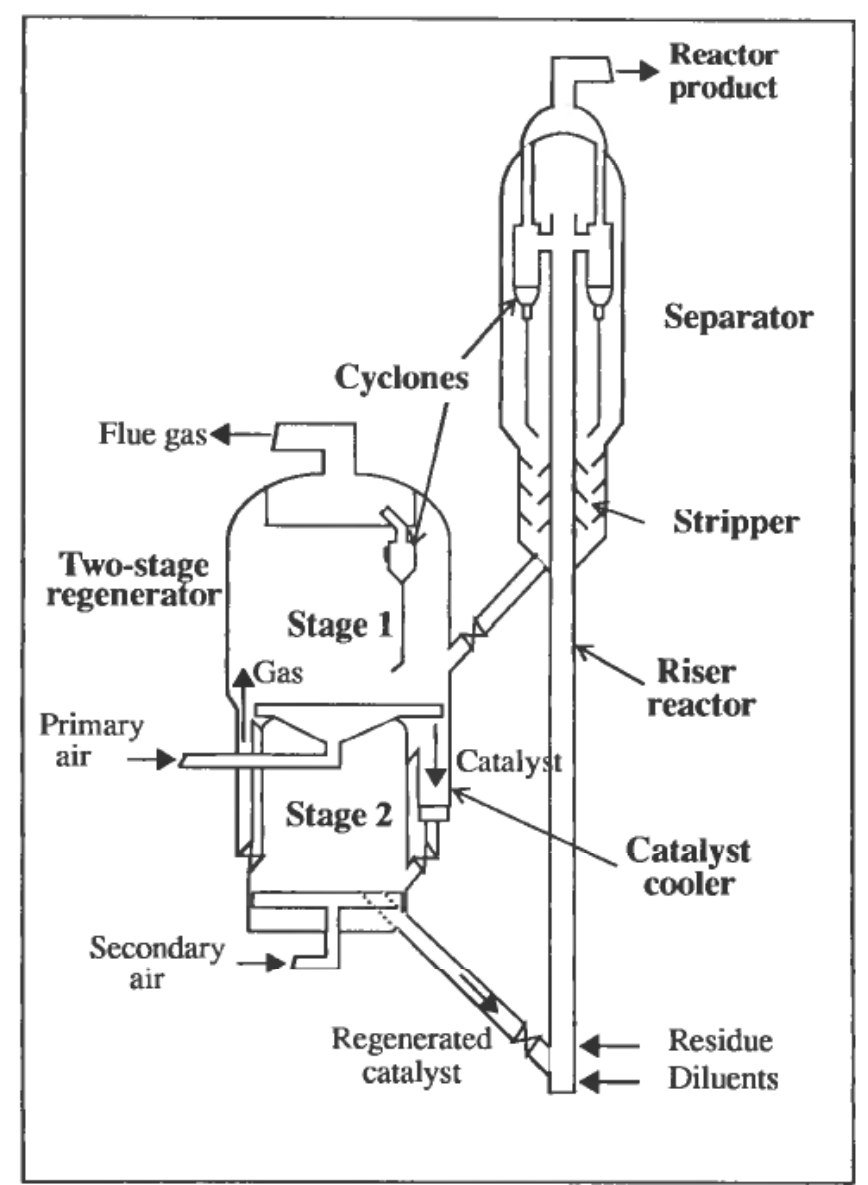

Figure 3. Schematic diagram of the RCC process.

\subsection{Process characteristics and RCC modeling}

Figure 3 highlights some of the design concepts that have been incorporated in the RCC process. This process differs somewhat from the conventional FCC unit. When it comes to the dynamic behaviour, the main difference is the two-stage regenerator and the catalyst cooler of the RCC unit. The first stage is operated at a low-temperature or conventional regeneration mode where there is excess coke on regenerated catalyst while the second stage is operated in a high-temperature regeneration mode, where there is excess oxygen. There is a dry environment in the second stage which means that the catalyst is completely regenerated (less than $0.05 \mathrm{wt} \%$ coke) with a minimum of hydrothermal deactivation.

Although the RCC hardware is more complicated than that of the FCC unit, this does not necessarily mean that it is more time consuming to develop a satisfactory RCC model. On the contrary, hardware details which move the process closer to idealistic performance, make simplifying modeling assumptions more valid. However it is well known that the regenerator exposes very different dynamics in the high and low temperature operational modes. Consequently the staged regenerator expose complex dynamics, and the RCC unit would have been very difficult to control if the catalyst cooler had been excluded. 

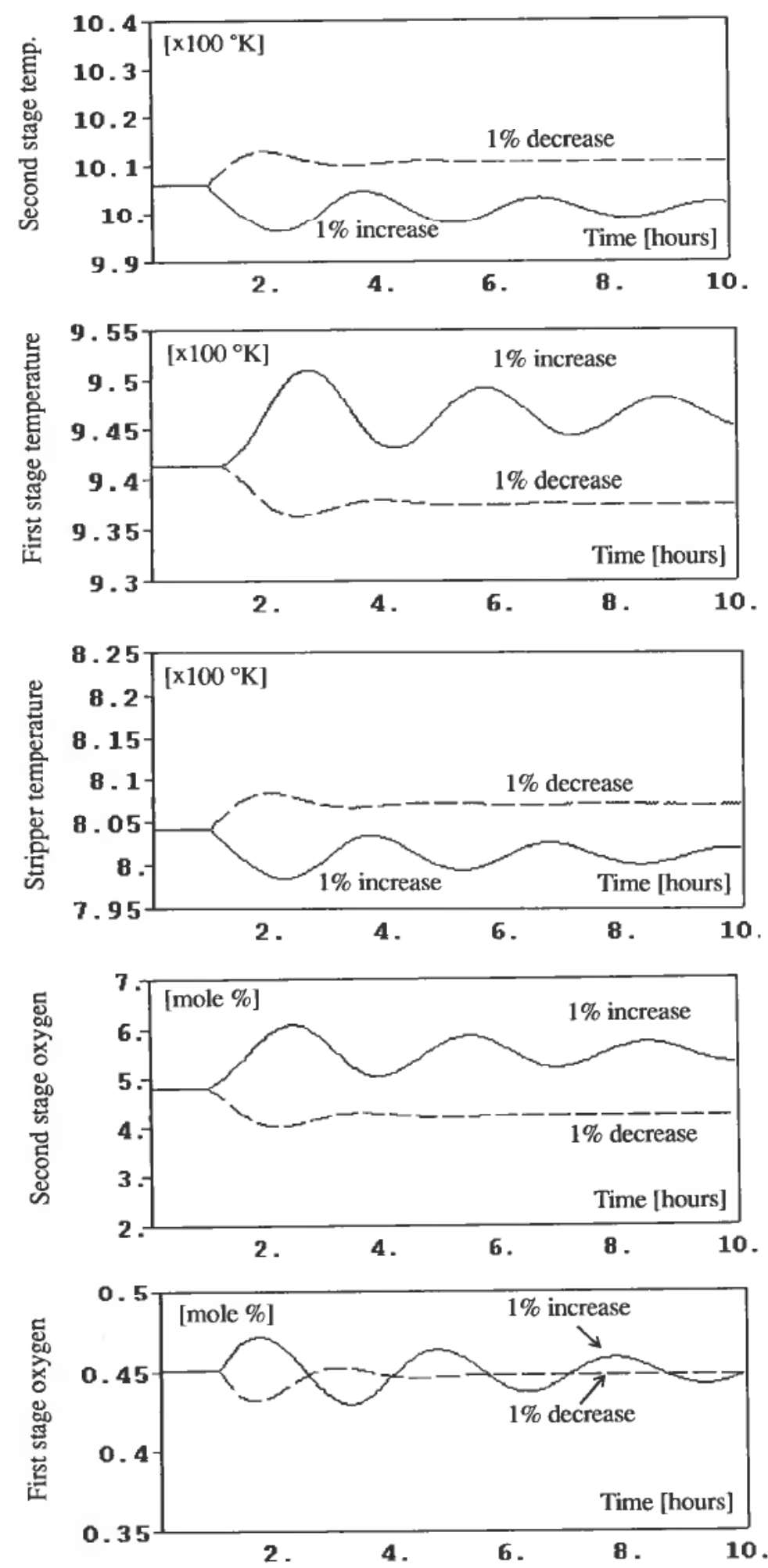

Figure 4. Step responses of $1 \%$ increase and decrease in the external catalyst cooling rate at $t=1$ hour. 
The RCC model presented by Ljungquist (1990) reflects the basic characteristics of the RCC dynamics. Some of the assumptions on which the model is based may be somewhat unrealistic, and the model has to be modified and slightly extended before it can be implemented in a predictive control scheme. Simulation studies have illustrated that the RCC process is highly nonlinear and that the dynamics is even more complex than that of the FCC process:

- There is a weakly damped resonance phenomenon whose damping ratio may vary more than one decade as the operational point changes.

- There are dominating nonminimum phase phenomena in the process.

Analysis of the RCC model shows that the complex dynamics of the RCC process is due to the strong interaction between the two regenerator stages. This interaction is reduced as the temperature difference increases, and the resulting process becomes easier to control.

In Fig. 4 the simulated responses of step changes in the catalyst cooling rate are examined. The responses demonstrate that the temperature difference between the two regenerator stages increases as the cooling rate decreases. Consequently, the process performance resulting from a higher cooling rate is degraded. Besides, these simulation experiments demonstrate that the catalyst cooling rate is a very powerful control variable.

\subsection{RCC control}

The operational flexibility in the RCC process makes this process easier to control than the FCC unit. The implemented RCC model, possibly with some minor modifications to account for neglected effects, is a useful tool to develop new RCC control strategies. One application is to study the pairing problem, if conventional PID controllers are to be used. Even though such control schemes may operate well at a given operation point, they will not take advantage of the inherent flexibility in the process. The reasons for this are the strong interactions and nonlinearities, which mean that neither the pairing nor the controller turning are optimal at another operation point. More advanced control schemes should therefore be considered. The high nonlinearity of the RCC process and partly known (sometimes predictable) changes given model parameters mean that a predictive control scheme should be based on a nonlinear state-space model.

\section{Conclusion}

In model-predictive control of processes with highly nonlinear dynamics and predominant constraints such as catalytic cracking processes, it is important that the model gives realistic predictions of important process variables over a relatively long horizon. The consequence of this is that the most sensible choice of model structure is the nonlinear state-space model. Moreover, a model-predictive controller which fully utilizes the potentials of this model structure should be used to maximize the profit. The SSPC meets these requirements.

\section{ACKNOWLEDGMENT}

This research has been sponsored by the Royal Norwegian Council for Scientific and Industrial Research (NTNF) through the MIP program. 


\section{REFERENCES}

Balchen, J. G., Ljungquist, D. and Strand, S. (1988a). Predictive Control Based Upon State Models. Proc. of the 1988 ACC, 3, 2174-2179.

Balchen, J. G., LuUngquist, D. and Strand, S. (1988b). State Space Predictive Control of a Multistage Electrometalurgical Process. Proc. of the IF AC Workshop on Model Based Process Control, Atlanta, Georgia, June 1988 (Pergamon Press, Oxford), 47-53.

Balchin, J. G., Luungeuist, D. and Strand, S., (1991). State Space Predictive Control. Chemical Engineering Science, 47, 987-807.

Balchen, J. G. and Mummé, K. I. (1988) Process Control: Structures and Applications (Van Nostrand Reinhold, New York) pp. 369-375.

Caldwell, J. M. and Dearwater, J. G. (1991) Model Predictive Control Applied to FCC Units. Preprints of CPC IV, Fourth International Conference on Chemical Process Control, Feb. 17-22 1991, South Padre Island, Texas.

Cutler, C. R. and RamaKer, B. L. (1979). Dynamic Matrix Control-a Computer Control Algorithm. AIChE 86th National Mtg, Houston, TX, Apr. 1979.

Garcia, C. E. and Morshedi, A . M. (1986). Quadratic Programming Solution of Dynamic Matrix Control (QDMC). Chem. Eng. Commun., 46, 73-87; also Proc. of the 1984 ACC, San Diego, CA.

Hemler, C. L., Lomas, D. A. and TAJBL, D. G. (1984). Residual Catalytic Cracking: Theory and Practice. Proc. Refin. Dep. Am. Pet. Inst., 63, 228-234.

Hомiк, K., SтіnснсомвE, M. and Whiтe, H. (1989). Multilayer Feedforward Networks are Universal Approximators. Neural Networks, 2, 359-366.

Huang, Z., Sapre, A. V. and Tsiligiannis, C. (1989). Dynamic Modelling and Control of Fluidized Catalytic Cracking Units. AIChE Annual Mtg., San Francisco Nov 1989, paper no. 22c.

JANG, S. S., JOSEPH, B. and MUKaI, H. (1987). On-line Optimization of Constrained Multivariable Chemical Processes. AIChE Journal, 33, no. 1, 26-35.

JoSEPH, B., JANG, S. S. and MuKAI, H. (1988). Integrated Model Based Control of Multivariable Nonlinear Systems. Proc of the IF AC Workshop on Model Based Process Control, Atlanta, Georgia, June 1988 (Pergamon Press, Oxford) 1989, pp. 121-128.

LeE, E. and Groves, F. R., Jr. (1985). Mathematical Model of the Fluidized 引ed Catalytic Cracking Plant. Trans. Soc. Comp. Sim., 2, 219-236.

LJUNGQUIST, D. (1990) Online Estimation in Nonlinear State-Space Models with Application to Catalytic Cracking. Dr. Ing. thesis. Report no. 90-89-W, Divison of Engineering Cybernetics, NTH, Trondheim.

McFarlane, R. C., Reineman, R. C., Bartee, J. F. and Georgakis, C. (1990). Dynamic Simulator for a Model IV Fluid Catalytic Cracking Unit. AIChE Annual Mtg., Chicago Nov. 1990.

Patwardhan, A. A., Rawlings, J. B. and Edgar, T. F. (1988). Model Predictive Control of Nonlinear Processes in the Presence of Constraints. AIChE Annual Mtg., Washington, D.C., Session 129, paper no. 129 b.

Poggio, T. and Girosi, F. (1990). Networks for Approximation and Learning. Proceedings of the IEEE, 78, 1481-1497.

Prett, D. M. and Gillette, R. D. (1979). Optimization and Constrained Multivariable Control of a Catalytic Cracking Unit. AIChE National Mtg., Houston, TX, April 1979; also Proc. Joint Automatic Control Conf., San Francisco, CA, 1980.

Richalet, J. A., Rault, A., Testud, J. D. and Papon, J. (1978). Model Predictive Heuristic Control: Applications to Industrial Processes. Automatica, 14, 413-428.

SCHMid, C. and BiEGLER, L. T. (1990) Application of Multistep Newton-type Controllers to Fluid Catalytic Cracking. Proc. of the 1990 ACC, 581-586.

Sistu, P. B. and BEQUETTE, B. W. (1990) Process Identification using Nonliner Programming Techniques. Proc. of the 1990 ACC, 1534-1539.

Strand, S. (1991) Dynamic Optimization in State-Space Predictive Control Schemes. Dr. Ing. thesis. Report no. 91-11-W, Division of Engineering Cybernetics, NTH, Trondheim.

UOP, U.S. Patent no. 4,849,091, 1989.

Zandona, O. J., Busch, L. E. and Hetringer, W. P., Jr. (1982) Reduced Crude Oil Processing with Ashland's RCC Process. Proc. Am. Pet Inst. Refin. Dep., 61, 207-215 